\title{
Fatal Cardiac Tamponade in Newly Diagnosed Systemic Sclerosis with Early Cutaneous Disease: A Case Report
}

\author{
Akintayo Richard Oluyinka ${ }^{*}$, Yusuf Idris Abiola², Adeyemi Olusola Ayodele ${ }^{3}$, \\ Akintayo Comfort Foluke ${ }^{4}$ \\ ${ }^{1,2,3}$ Department of Medicine, University of Ilorin Teaching Hospital, PMB 1459, Ilorin, Nigeria. \\ 4Department of Family Medicine, LAUTECH Teaching Hospital, Ogbomosho, Nigeria.
}

Received: March 3, 2017; Accepted: August 23, 2017; Published: September 05, 2017

*Corresponding author: Akintayo Richard Oluyinka, Department of Medicine, University of Ilorin Teaching Hospital, PMB 1459, Ilorin, Nigeria, Tele: +2348038433347; E-mail: richocounlimited@gmail.com

\begin{abstract}
Background:Systemic Sclerosis (SSc) is a rare systemic autoimmune disease with a potential to affect most organ systems of the body. Symptomatic pericardial effusion is rarely reported as the first reason for hospital visit in a case of undiagnosed SSc.

Case summary: A 53year old Nigerian woman was admitted with dyspnea, recurrent Raynaud's phenomenon and scanty skin sclerosis over the dorsum of the hands and the upper back. Echocardiography revealed moderate pericardial effusion and severe pulmonary hypertension. She had positive antinuclear antibody and anti-Scl-70. A diagnosis of systemic sclerosis was made and she was treated with low dose prednisolone, frusemide and tadalafil. She recovered with complete resolution of the pleural effusion over 2 weeks but she presented to the emergency unit a month later in cardiac tamponade. Repeat echocardiography showed re-accumulated pericardial effusion with a compressed left ventricle. She suffered a cardiac arrest before a pericardiocentesis could be done and she could not be revived.
\end{abstract}

Conclusion: Early SSc should be considered while investigating a case of pericardial effusion of unidentified cause. These cases may be more prone to tamponade even before a large amount of pericardial effusion accumulates.

Keywords: Systemic sclerosis; Pericarditis; Cardiac tamponade; Raynaud's disease; Antinuclear antibody.

\section{Introduction}

Systemic Sclerosis (SSc) is a rare systemic autoimmune rheumatic disease with a potential to affect most organ systems of the body. The clinically heterogenous disease manifests in genetically susceptible individuals with a diverse pattern of symptomatology [1]. Hence, patients with SSc represent a host of different manners of disease onset, presentation, course and prognosis. The presence of cardiac involvement, like other major organ affectation, in SSc portends a poor outlook. Direct cardiac involvement in SSc may affect the pericardium, myocardium, conduction system, or rarely the heart valves [2]. Autopsy studies have revealed that the prevalence of pericardial involvement in SSc is high but rarely are patients symptomatic for it [3]. It has been suggested that the frequency of pericardial disease may be higher in patients with limited cutaneous SSc than in those with diffuse skin disease [2]. Pericardial abnormalities described in post-mortem studies of SSc include fibrinous pericarditis, fibrous pericarditis, pericardial adhesions, and pericardial effusions [4]. In sub-Saharan Africa, infectious aetiologies are the usual suspect in most cases of unexplained pericardial effusion and tuberculosis is often blamed for many cases of unconfirmed cause. Moreover, early presentation with recurrent symptomatic pericardial effusion has not been reported in West African SSc patients. We report a case of recurrent symptomatic pericardial effusions in a case of early SSc.

\section{Case Presentation}

A 53-year old female teacher was admitted on account of a week history of progressive breathlessness of insidious onset. There was dyspnea on mild to moderate exertion. She had a minimal chest pain which had lasted several days and had disappeared a few days before presentation. The pain was not severe enough to prevent her from her usual activities but the dyspnea limited her effort tolerance. There was no history of paroxysmal nocturnal dyspnea but there was orthopnea. There was no fever, night sweats or weight loss. She however had a six-month history of recurrent attacks of mild Raynaud's phenomenon for which she had never seen a doctor. She had not noticed any skin changes or dysphagia. There was no history of photosensitivity, oral ulcers, sicca symptoms or muscle weakness. She had no history of smoking or alcohol intake.

She was afebrile, not pale, and she had bilateral pitting pedal edema. Pulse rate was 108 beats per minute, regular and 
of moderate volume. The blood pressure was 110/70 mmHg. Jugular venous pressure was visibly raised and the cardiac apex was difficult to palpate. Heart sounds were first and second with a diastolic murmur over the pulmonary area. Minimal skin tightening was demonstrable over the dorsum of the fingers and this extended to just proximal to the metacarpophalangeal joints bilaterally. There were no pigmentary changes over these areas. She had an area of multiple hypopigmented macules in her upper back. There was some skin thickening over this area as well. Other skin regions were normal.

ECG showed sinus tachycardia and widespread low voltages. Sp02 was $96 \%$ in room air. No fibrotic or cavitatory lesions were seen in the lung fields on chest X-ray. However, the cardiothoracic ratio was 0.6. Echocardiography showed moderate pericardial effusion, grossly enlarged right atrium and right ventricle, the left ventricular chamber was normal in size and contractility with Ejection fraction of $75 \%$ measured by Simpson's method. There was severe pulmonary hypertension with an estimated Pulmonary Artery Systolic Pressure (PASP) of $75 \mathrm{mmHg}$. Tricuspid and pulmonary valvular regurgitations were also noted. A previous resting echo study done 10 months earlier was normal. Tuberculosis was excluded by negative mantoux and quantiFERON-TB Gold tests. Urinalysis as well as electrolytes, urea and creatinine were normal. Viral screening was negative for HIV, Hepatitis B and Hepatitis C. Antinuclear antibody (ANA) and Anti-Scl-70 were positive. The ANA titre was 1:320.

A diagnosis of systemic sclerosis with pericarditis and severe pulmonary hypertension was made. She was commenced on prednisolone $5 \mathrm{mg}$ daily, frusemide $40 \mathrm{mg}$ twice daily and tadalafil $20 \mathrm{mg}$ daily. The pericardial effusion gradually resolved over the following 2 weeks and she was discharged in a stable clinical state. A follow up visit was scheduled for 2 weeks. She presented a month later via the emergency unit in respiratory distress with features of circulatory shock and a prominent jugular venous pulsation reaching the angle of the jaw. Echocardiography demonstrated moderate pericardial effusion which was slightly more than the one found in the first study during the initial admission. The left ventricle was compressed between the enlarged right ventricle and the unyielding thickened pericardium and effusion. The left ventricular cavity was reduced to a narrow chamber (Figure 1). She suffered a cardiac arrest before an emergency percutaneous drainage could be done and all resuscitative attempts proved abortive.

\section{Discussion}

SSc ranks highest among connective tissue diseases with a tendency to have some pericardial involvement while the lowest frequency of pericardial disease is probably in the idiopathic inflammatory myopathies [5]. However, SSc may not rank as high among other connective tissue diseases when the frequencies of symptomatic pericardial disease are considered strictly. The finding of mild chronic pericardial effusion is fairly common in SSc and patients are rarely symptomatic for it. The development of cardiac tamponade in these patients is even rarer [6]. Therefore, a previously undiagnosed patient with early cutaneous changes of SSc who develops cardiac tamponade constitutes a potential complexity of diagnostic considerations. Up to $16 \%$ of patients will be clinically symptomatic for pericardial disease at some point in the course of their disease. This is much less than necropsy-demonstrated pericardial involvement in up to $70 \%$ [4]. This probably represents the usual pattern of more advanced or severe cases in post-mortem studies.

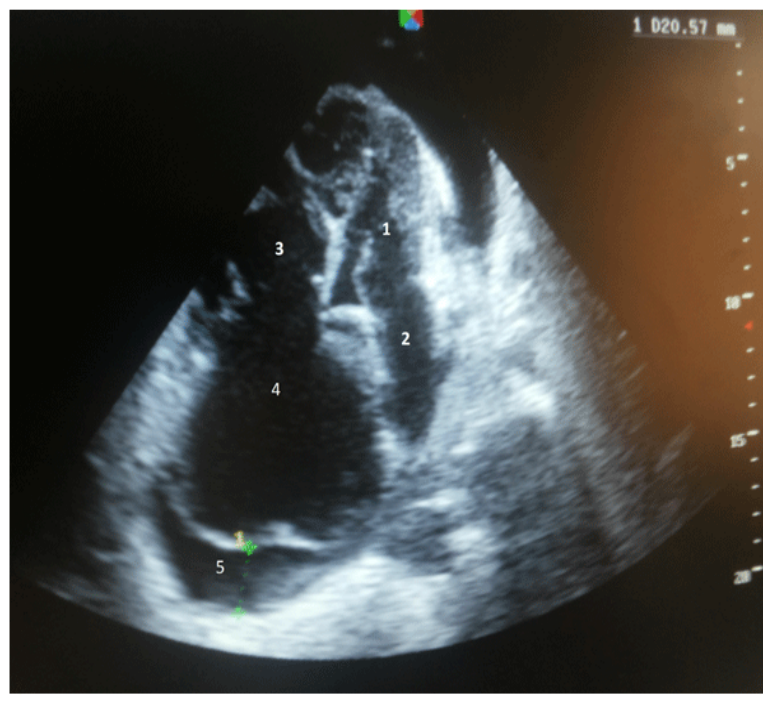

Figure 1: Echocardiogram showing the left ventricle [1], left atrium [2] and the dilated right ventricle [3] and right atrium [4]. Moderate pleural effusion [5] is also demonstrated.

Scanty cases of SSc presenting first with symptomatic pericardial effusion have been described $[3,7,8]$. Arriving at the root diagnosis of such cases in most centres in West Africa could be delayed. This is partly due to the erstwhile belief that connective tissue diseases are very rare in sub-Saharan Africa [9]. As such many cases of SSc may not be recognized until cutaneous manifestations are florid. Also, infectious agents particularly tuberculosis is often thought to be responsible for most cases of unexplained pericardial disease even in the face of lacking microbiologic evidence.

Fibrotic changes of the pericardium are common in SSc, although excess amounts of mast cells are not found [10]. Thus patients may be prone to cardiac tamponade before the accumulation of large volumes of pericardial effusion. This subnormal distensibility of the pericardium probably predisposes SSc with pericardial effusions to a higher likelihood of having a tamponade [6]. It is therefore for this reason that Langley et al recommended looking out for the onset of a tamponade and being prepared to treat it especially if there is the occurrence of a sudden cardiovascular collapse [5]. Conversely, the experience from John Hopkins is that SSc with significant pulmonary hypertension is often associated with a poor outcome if a percutaneous drainage of pericardial effusion is performed [4]. Low dose oral 
prednisolone was commenced during the first presentation on suspicion of an inflammatory basis for the effusion. This conservative approach seemed adequate for the first episode but in the face of an emergent circulatory compromise, the need for effusion drainage is undeniable.

Our patient had severe pulmonary hypertension on echocardiography with associated severe regurgitations of the pulmonary and tricuspid valves. A right heart catheterization could not be done because the facility is not available in our setting. Pulmonary arterial hypertension $(\mathrm{PAH})$ is associated with a $50 \%$ three-year mortality rate in SSc and it is found in up to $12 \%$ of all patients with SSc $[11,12]$. There are conflicting reports on the more prevalent disease between SSc-associated $\mathrm{PAH}$ and idiopathic PAH with some studies suggesting that the former may be as many as four times the latter and others finding the opposite $[13,14]$. The multisystemic nature of SSc often implies an aggregation of multiple organ diseases. This is likely responsible for a threefold increase in the risk of death as compared with patients with idiopathic PAH. Moreover, for unknown reasons, conventional treatments for idiopathic $\mathrm{PAH}$ are less effective in PAH in SSc [11]. Primary myocardial disease, among other factors, is associated with the presence of various degrees of diastolic dysfunction in some patients with SSc [15].

In conclusion, early SSc is an important differential to consider while investigating a case of pericardial effusion of unidentified cause. Also, these patients may have ongoing PAH, diastolic dysfunction or fibrotic pericardial disease predisposing them to cardiovascular compromise from relatively moderate volumes of pericardial effusion.

\section{References}

1. Salazar G, Mayes MD. Genetics, epigenetics, and genomics of systemic sclerosis. Rheum Dis Clin North Am. 2015;41:345-66. doi: 10.1016/j. rdc.2015.04.001.

2. Lambova S. Cardiac manifestations in systemic sclerosis. World J Cardiol. 2014;6:993-1005. doi: 10.4330/wjc.v6.i9.993.

3. Peronato G, De Sandre P, Podswiadek M, Arfiero S, Bozzola L. cardiac tamponade preceding skin involvement in progressive systemic sclerosis. Reumatismo. 2002;54(3):257-60.
4. Champion HC. The heart in scleroderma. Rheum Dis Clin North Am. 2008;34(1):181-viii. doi: 10.1016/j.rdc.2007.12.002.

5. Langley RL, Treadwell EL. Cardiac tamponade and pericardial disorders in connective tissue diseases: Case report and literature review. J Natl Med Assoc. 1994;86(2):149-53.

6. Subramanian SR, Akram R, Velayati A, Chadow H. New development of cardiac tamponade on underlying effusive-constrictive pericarditis: An uncommon initial presentation of scleroderma. BMJ Case Rep 2013;2013. doi: 10.1136/bcr-2013-010254.

7. Itolikar SM, Salagre S, Phatak S, Itolikar M. Recurrent cardiac tamponade in a young woman. BMJ Case Rep 2013;2013. doi: 10.1136/bcr-2013-201472.

8. Perez-Bocanegra C, Fonollosa V, Simeon CP, Candell J, Solans R, et al. Pericardial tamponade preceding cutaneous involvement in systemic sclerosis. Ann Rheum Dis. 1995;54:687-688.

9. Adelowo 0, Bello M. Systemic autoimmune diseases: Not so rare in black africans. State of immunity. 2014;9:74.

10. Byers RJ, Marshall DA, Freemont AJ. Pericardial involvement in systemic sclerosis. Ann Rheum Dis. 1997; 56(6): 393-394.

11. Chaisson NF, Hassoun PM. Systemic sclerosis-associated pulmonary arterial hypertension. Chest. 2013;144: 1346-1356. doi: 10.1378/ chest.12-2396.

12. Avouac J, Airo P, Meune C, Beretta L, Dieude P, et al. Prevalence of pulmonary hypertension in systemic sclerosis in european caucasians and metaanalysis of 5 studies. J Rheumatol 2010;37(11):2290-2298. doi: 10.3899/jrheum.100245.

13. Gaine SP, Rubin LJ. Primary pulmonary hypertension. Lancet. 1998;352:719-725.

14. Badesch DB, Raskob GE, Elliott CG, Krichman AM, Farber HW, et al. Pulmonary arterial hypertension: Baseline characteristics from the reveal registry. Chest. 2010;137(2):376-387. doi: 10.1378/ chest.09-1140.

15. Meune C, Avouac J, Wahbi K, Cabanes L, Wipff J, et al. Cardiac involvement in systemic sclerosis assessed by tissue-doppler echocardiography during routine care: A controlled study of 100 consecutive patients. Arthritis Rheum. 2008;58(6):1803-1809. doi: 10.1002/art.23463. 